\title{
Extending SSM to MIPv6 - problems, solutions and improvements
}

\author{
Thomas C. Schmidt ${ }^{1,2}$ and Matthias Wählisch ${ }^{2}$ \\ ${ }^{1}$ HAW Hamburg, Department Informatik, Berliner Tor 7, 20099 Hamburg, Germany \\ ${ }^{2}$ FHTW Berlin, Hochschulrechenzentrum, Treskowallee 8, 10318 Berlin, Germany \\ e-mail: $\{$ schmidt|mw\}@fhtw-berlin.de
}

\begin{abstract}
Multicast data delivery carries distinct importance in mobile wireless environments, where bandwidth is limited and transmission channels are shared between users. The development of mobile multicast protocols consequently exhibits emerging interest. As the common approach of Any Source Multicast distribution is burdened with intricate routing procedures, the spread of multicast-enabled network infrastructure remains hesitant. It is widely believed that simpler mechanisms for group distribution in Source Specific Multicast (SSM) will lead to a pervasive dissemination of multi-cast infrastructure and services. However, SSM is designed for the a priori known and changeless addresses of multicast sources and thus withstands any easy extension to mobility. This paper reviews the state-of-the-art in current work to extend SSM to Mobile IPv6 networks. The principle conceptual problems are discussed and analysed. Propositions for improvement and possible directions to proceed further in SSM source mobility are presented.
\end{abstract}

Key words: mobile IPv6, source specific multicast, SSM source mobility, routing

\section{INTRODUCTION}

The distribution of Internet traffic to groups of receivers is of increasing interest to all those, who consider the Internet ready to integrate synchronous media services. Voice and video conferencing today place the demand for serverless communication support within groups of manageable sizes [1]. In the near future, broadcast-type applications may require media data distribution to groups too large to be managed by any server-based system. IP multicasting may soon turn out to be the only feasible technique to be considered.

The next generation Internet will need to provide seamless support of mobility. Mobile devices today can be seen as one of the major driving forces for communication and multimedia data transmission. Cellular phones and portable paddles are expected to carry individual Internet addresses soon, as available from IPv6 address space, and to operate mobile IPv6 (MIPv6) [2]. It is the vision that "IPv6 will be pervasive and prevalent across all digital device communications and augurs well for mobility and wireless access on the Internet" [3]. IP multicasting will be of particular importance to mobile environments, where users commonly share frequency bands of limited capacities. Up until now, the mature support of multicast mobility within the Internet remains unseen - we all are thus urged to work out solutions for bridging that gap.

Source Specific Multicast (SSM) [4, 5] is considered a promising improvement of group distribution techniques. In contrast to Any Source Multicast (ASM) [6] routing, shortestpath $(\mathrm{S}, \mathrm{G})$ multicast source trees are immediately constructed from client subscription without utilising shared trees or rendezvous points. Routing is initiated from clients joining ( $\mathrm{S}$, G) channels instead of $(*, \mathrm{G})$ groups, presupposing á priori known addresses of the multicast sources. There are many applications providing out-of-band knowledge of source addresses, e.g., SIP-based [7] conferences or broadcasting-type distributions. Source addresses may also be acquired by administrative channels such as SAP/SDR or a Web page. Furthermore, there are promising attempts to develop source address discovery mechanisms on the Internet layer [8,9].

Simplified routing in SSM relies on source identifications, which invalidate when source addresses change under mobility. Fundamentally, SSM was designed for changeless multicast source addresses, leaving SSM source mobility as a major open problem. Source addresses in SSM packets carry the dual meaning of being source-group identifiers on the one hand and routing locators on the other hand. In the context of mobility, two principal problems need to be considered: At first, a multicast listener willing to subscribe to an $(\mathrm{S}, \mathrm{G})$ channel needs to account for the current location of the mobile source. Further on, in the occurrence of movement, routing is required to transform any $(S, G)$ state into $\left(S^{\prime}, G\right)$, while listeners continue to receive multicast data streams.

In this paper, we will present and analyse the current state of concepts for mobile multicast sources in general, and for SSM mobility in detail. We will deduce, why immediate transitions of ASM mobility concepts to SSM do not hold. Moreover we will identify conceptual regions for improvement and propose ideas for the development of new SSM mobility schemes. The following chapter is dedicated to discussing source mobility in ASM and SSM, providing and overview of current approaches. In chapter 3 we will introduce new ideas for future consideration in SSM mobility solutions. Finally, conclusions and an outlook follow in chapter 4. 


\section{MOBILE MULTICAST SOURCES}

\subsection{Mobility in Any Source Multicast}

Multicast group communication raises quite distinctive aspects within a mobility-aware Internet infrastructure: On the one hand, Multicast routing itself supports dynamic route configuration, as members may join and leave ongoing group communication over time. On the other hand, multicast group membership management and routing procedures are intricate and too slow to function smoothly for mobile users. In addition, multicast imposes a special focus on source addresses. Applications commonly identify contributing streams through source addresses, which must not change during sessions, and routing paths in most protocols are chosen from destination to source. In general, the roles of multicast senders and receivers are quite distinct. While a client initiates a local multicast tree branch, the source may form the root of an entire source tree. Lasting, at mos, seconds, branch construction only affects selected receivers, whereas re-erecting a source-based tree may interrupt the entire session for some minutes. Hence multicast mobility at the sender side poses the more delicate problem.

Three approaches to mobility in Any Source Multicast are common:

Bi-directional Tunnelling guides the mobile node to tunnel all multicast data via its home agent. This principle multicast solution from [2] hides all movement and results in static multicast trees. It may be employed transparently by mobile multi-cast sources, on the principle of triangular routing and possibly significant performance degradations due to widely spanned data tunnels.

Remote Subscription forces the mobile node to re-initiate multicast distribution subsequent to handover, using its current Care-of Address. This approach of tree discontinuation relies on multicast dynamics to adapt to network changes. It not only results in rigorous service disruption, but leads to mobility driven changes of source addresses, and thus disregards session persistence under multicast source mobility.

Agent-based solutions attempt to balance between the previous two mechanisms. Static agents typically act as local tunnelling proxies, allowing for some inter-agent handover while the mobile node moves away. A decelerated inter-tree handover, i.e., tree walking, will be the outcome of agent-based multicast mobility, where some extra effort is needed to sustain session persistence through address transparency of mobile sources.

While significant work has been invested in protocol design for mobile multicast receivers, only limited effort has been dedicated to multicast source mobility [10]. An agent-based solution to multicast mobility for receivers and senders has been presented to the IETF in [11], which concurrently (without bicasting) initiates a new distribution tree and terminates the previous on timeout.

The intricacy specific to multicast source mobility derives from the principle of decoupling of source and receivers: A multicast source submits data to a group of unknown receivers, thus operating without any feedback channel ${ }^{1}$. It neither has means to inquire about the properties of its delivery trees, nor will it be able to learn about the state of its receivers. In the event of an inter-tree handover, a mobile multicast is source vulnerable to loss of receivers without taking notice. Interesting ideas of re-using modified trees have been introduced recently. The authors of [13] present a root extension of DVMRP shortest path trees for mobile sources, in [14] a unicast backbone distribution of packets is suggested to account for the problem of interdomain source activation.

It should be noted that multicast source mobility always places a heavy burden onto the routing infrastructure. The employment of proxy schemes to reduce handover frequencies are advisable and of significant effect [15].

\subsection{Mobility in Source Specific Multicast}

Source mobility for SSM packet distribution introduces an additional conceptual complexity. As a listener is subscribed to an $(\mathrm{S}, \mathrm{G})$ channel membership, and as routers have established an (S, G)-state shortest path tree rooted at source $S$, any change of source addresses under mobility requests state updates at all routers and all receivers. A moving source would have to update its change of CoA with all listeners, which subsequently had to subscribe anew and initiate corresponding source-specific trees. As the principle multicast decoupling of a sender from its receivers likewise holds for SSM, the need for client update becomes a severe problem. It becomes evident that ASM mobility approaches, aside from bi-directional tunnelling, do not easily extend to SSM. While any remote subscription technique will simply not reach group receivers, agent-based approaches will fail, since SSM inter-tree handovers cannot be solely initiated by a multicast source. To understand the latter, recall that a change of distribution tree requires clients to actively subscribe to the new tree.

As in ASM, source mobility sessions need to be preserved while source addresses change. To account for the latter, Thaler [16] proposes the employment of Binding Caches and obtainment of source address transparency via duplicate addressing as in unicast MIPv6. Current addresses of the mobile SSM source are to be announced via an additional Home Agent-based tree. Any client subscribing to (HoA, G) will thus learn $(\mathrm{CoA}, \mathrm{G})$, initially and during ongoing sessions, as receivers following this SSM mobility scheme are required to remain members of the $(\mathrm{HoA}, \mathrm{G})$ address announcement channel. Address announcements are periodically sent down this administrative multicast tree, which accounts for additional mobility overhead. On handover, clients have to await the new care-of address announcement and re-subscribe to the new $(\mathrm{nCoA}, \mathrm{G})$ channel. Source-tree handovers in this proposed protocol thus remain far too slow to be considered seamless. As multi-cast sessions remain unsecured, the simple use of multicast Binding Updates may also cause considerable security flaws

${ }^{2}$ Previous work on Multicast Source Notification of Interest Protocol [12] has been terminated by IETF. 
with the possible result of unicast sessions highjacked by the multicasting. The latter, of course, can be healed by using a separate multicast binding cache.

Jelger and Noel [17] suggest mobile SSM handover improvements by employing anchor points within the source network, causing continuous data reception during client- initiated handovers. Their 'tree walking' approach can be understood as a fairly direct transformation of traditional agent-based solutions in ASM mobility: In the event of a handover, the mobile source reconnects with the previous anchor point and distributes a new "SSM-Source Handover Notification" binding the update option. On reception of this binding update, listeners re-subscribe to the address channel of the new source, while multicast streams are continuously bi-casted down the previous distribution tree. Initial addresses are advertised in (HoA, CoA, G) triples via SDR/SAP ASM announcements.

In applying an ASM-type agent-based approach, the authors disregard the consequences of the decoupling principle for SSM.

\section{NEW DIRECTIONS FOR SSM SOURCE MOBILITY}

\subsection{Initial discovery of the mobile source}

Any SSM networking scheme, robust under source mobility, must provide a transparent multicast session layer while operating changes of address with routers and decoupled receivers. Multicast source address persistence may be inherited from unicast mobility: A mobile multicast source (MS) away from home will transmit unencapsulated data to a group using its HoA on the application layer and its current CoA on the Internet layer, just as unicast packets are transmitted by MIPv6. Likewise, data packets will carry an MIPv6 mobility destination option header to pass HoA as the source identifier to the application layer at the receiver side.

For primary subscription, a multicast receiver needs to be aware of the MS's current care-of address for initiating a (CoA, G) delivery channel. Discovery of the current address still can be

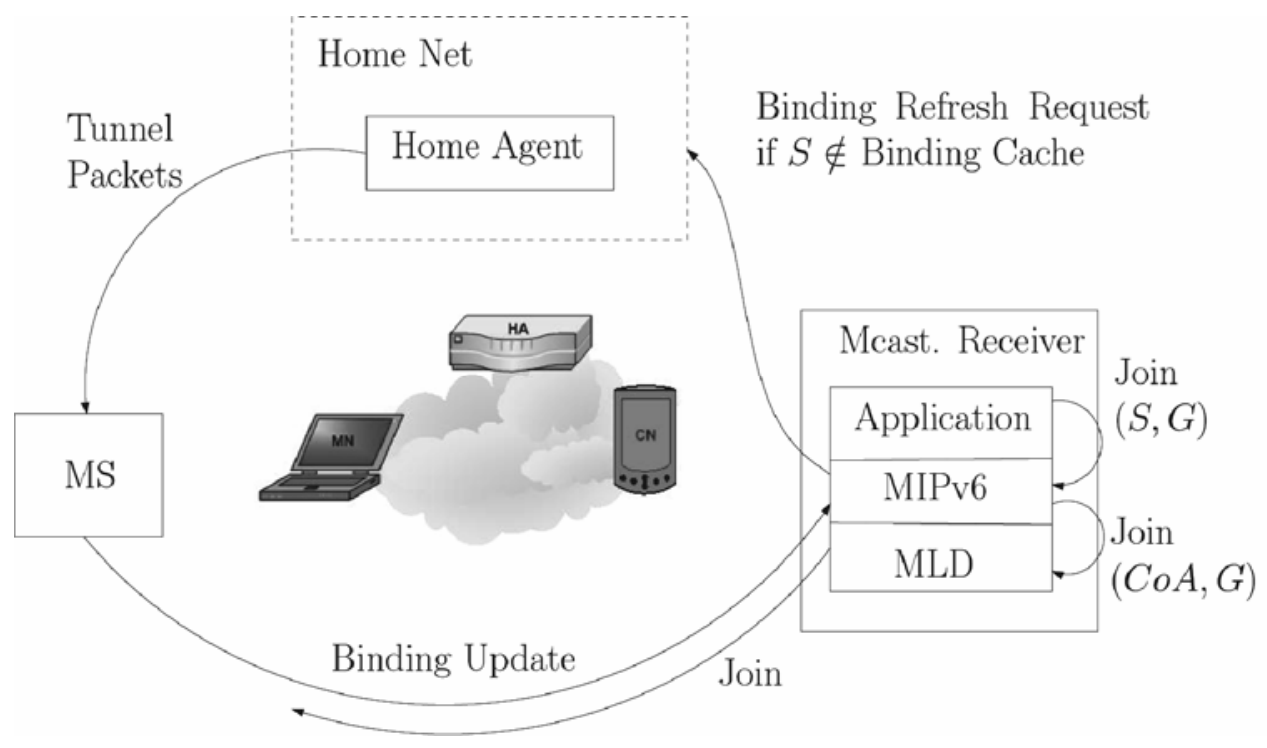

Fig. 1. Initial care-of address discovery by receiver's mobile IPv6 stack

Bi-casting to the previous SSM tree cannot be terminated until all receivers have established membership of the new distribution tree. In addition, SSM inter-tree handovers cannot be revoked, while receiver-oriented handovers in SSM will lead to an unforeseeable temporal progress. In case of rapid movement, a source operating the handover scheme of [17] is thus left with an unlimited number of previous delivery channels to be fed simultaneously.

Current approaches to SSM source mobility as reviewed above demonstrate the potentials and shortfalls achieved, when common ASM techniques are extended. As for SSM all IP nodes, i.e., source, routers and receivers, are involved in mobility operations, we believe that new ideas are needed to address the topic. Some novel attempts are presented in the following chapter. transparently provided by an extended MIPv6 stack in the following way (compare Fig. 1):

Applications, which remain unaware of mobility, will join an $(S, G)$ channel, where $S$ represents the home address of the SSM sender. The mobile multicast-aware MIPv6 stack will receive the join request and lookup $S$ in its binding cache. If present, a corresponding map entry will lead to the correct $(\mathrm{CoA}, \mathrm{G})$ subscription. As in general a binding cache entry cannot be presupposed, the mobility stack will have to inquire on the currently valid source address. This may be achieved by submitting a binding refresh request (s. [2]) to the home address and will lead to a valid binding cache entry. Thereafter, the multicast receiver will be able to join the multicast session, which appears to be a (HoA, G) channel to the session transport layer. 


\subsection{Tree morphing - multicast routing adaptive to source mobility}

In this section, we will introduce a new concept of multicast routing, adaptive to source mobility. We start from the observation that SSM receivers need to actively handover to any newly initiated distribution tree, which by the decoupling principle cannot be controlled by the source alone. For SSM routing, it is therefore preferable, not to change, but to re-use and modify existing source-based trees. Transforming trees, though, will require routers to identify multicast streams by means of the logical source identifier, the permanent HoA. Maintaining (CoA, G, HoA) address-triples in router states will enable all nodes to simultaneously identify (HoA, G) - based group membership and (CoA, G) - based tree topology. multicast Router (pDR) to the next Designated Router (nDR). Subsequent to handover, it will immediately continue to deliver data along an extension of its previous source tree. Delivery is done by elongating the root of the previous tree from pDR to nDR (Fig. 2). This extension is achieved through a state update message, carried in a Hop-by-Hop option header and sentto the multicast destination address using source routing through pDR. All routers along the path, located at root elongation or previous delivery tree, thereby will learn MS's new CoA and implement appropriate forwarding states.

Routers on this extended tree will use RPF checks to discover potential short cuts. Registering nCoA as source address, those routers, which receive the state update via the topologically incorrect interface, will submit a join in

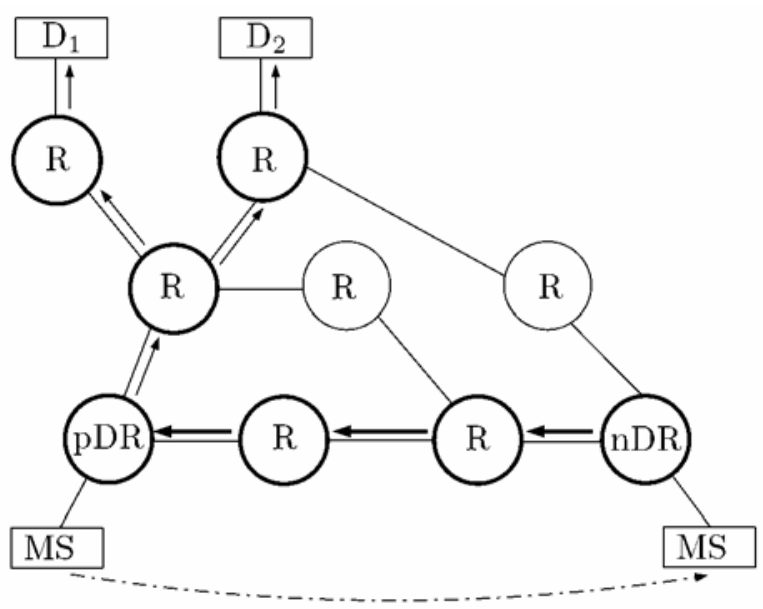

Fig. 2. Elongation of the tree root
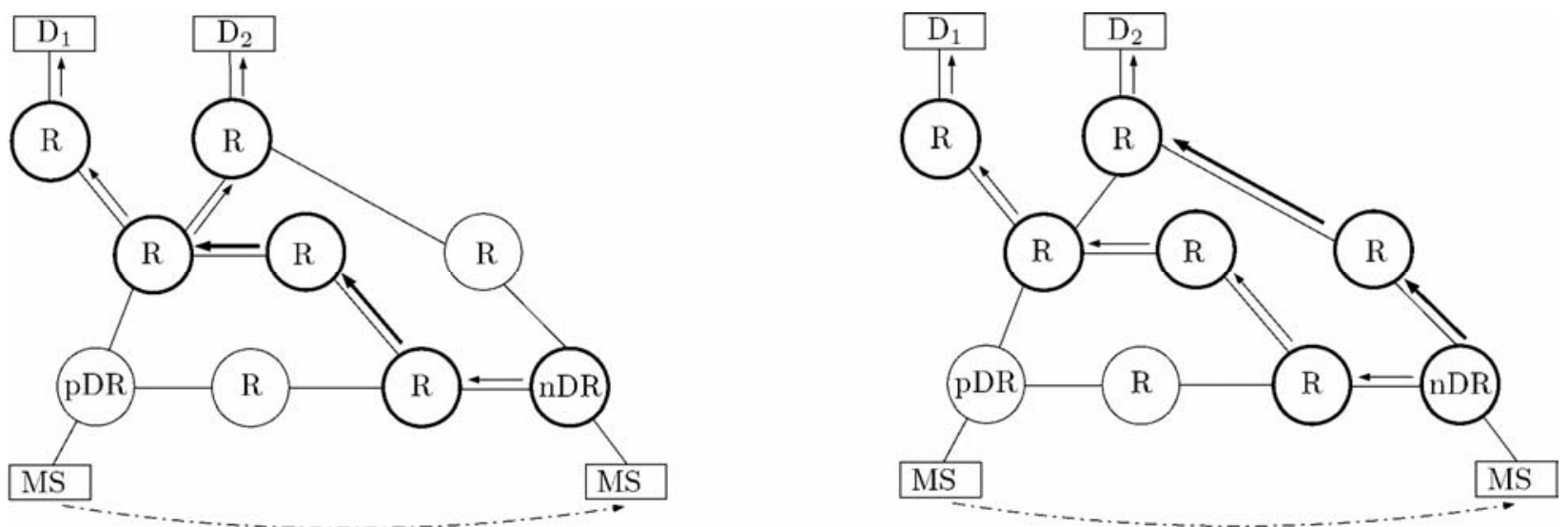

Fig. 3. Morphing states

When moving to a new point of attachment, the MS will alter its address from previous $\mathrm{CoA}(\mathrm{pCoA})$ to new $\mathrm{CoA}$ (nCoA) and eventually change from its previous Designated the direction of a new shortest-path tree and prune the old tree membership, as soon as data arrives. All other routers will simply overwrite their (pCoA, G) state with (nCoA, G). 
Thereby all parts of the previous delivery tree, which coincide with the new shortest-path tree, are re-used. Only branches of the new shortest-path tree, which have not previously been established, need to be constructed. In this way the previous shortest-path tree will be morphed into the next shortest-path tree as shown in Fig. 3.

Note that this algorithm does not require data encapsulation at any stage. It is not built upon a specific multicast routing protocol, but will require the following functional mechanisms compliant with current protocols such as PIMSM [18]:

- Outgoing router interfaces need to maintain $(S, G)$ states to denote their partition in the distribution tree. These states will be extended to include the Home Address identifier ( $S$, G, HoA);

- Routers need the ability to explicitly join an (S, G) state;

- Routers need the ability to explicitly prune an $(\mathrm{S}, \mathrm{G})$ state. Alternatively, but with lower efficiency, routing states may time out;

- Finally, the computation of standard Reverse Path Forwarding (RPF) check is used.

For the details of the signalling and routing protocol extensions under SSM mobility we refer the reader to [19].

\section{CONCLUSIONS AND OUTLOOK}

Mobile multimedia services are one of the major driving forces, but also a severe challenge for the Internet infrastructure today. In this paper we discussed multicast source mobility from the perspective of Source Specific Multicast. The principle conceptual difficulties have been derived and the current state-of-the-art has been evaluated. Initial ideas for new mobile SSM communication and routing schemes were presented, as they may serve as a starting point for revitalising the research on practically feasible solutions to this intricate field of development.

In future work we will optimise the details for the tree morphing, quantify its characteristic measures and simulate its overall behaviour. A formulation of a corresponding security layer will be on schedule, as well.

\section{References}

[1] T. C. Schmidt, M. Wählisch, H. L. Cycon, M. Palkow, Global serverless videoconferencing over IP, Future Generation Computer Systems, 19, 2, pp. 219-227, February 2003.

[2] D. B. Johnson, C. Perkins, J. Arkko, Mobility Support in IPv6, IETF, RFC 3775, June 2004.

[3] G. Kurup and Y. A. Sekercioglu, Source Specific Multicast (SSM) for MIPv6: A Survey of Current State of Standardisation and Research, in Proceedings of Australian Telecommunications, Networks and Applications Conference (ATNAC 2003), Melbourne, December 2003.
[4] S. Bhattacharyya, An Overview of Source-Specific Multicast (SSM), IETF, RFC 3569, July 2003.

[5] H. Holbrook and B. Cain, Source-Specific Multicast for IP, IETF, Internet Draft - work in progress 06, September 2004. [Online]. Available: ftp.rfc-editor.org/in-notes/ internetdrafts/draft-ietf-ssm-arch-06.txt

[6] S. E. Deering, Host Extensions for IP Multicasting, IETF, RFC 1112, Aug. 1989.

[7] J. Rosenberg, H. Schulzrinne, G. Camarillo, A. Johnston, J. Peterson, R. Sparks, M. Handley, E. Schooler, SIP: Session Initiation Protocol, IETF, RFC 3261, June 2002.

[8] S. Venaas and T. Chown, Source Specific Multicast (SSM) with IPv6, in Proceedings of the International Symposium on Applications and the Internet (SAINT 2005), Trento, February 2005, pp. 64-67.

[9] R. Lehtonen, S. Venaas, M. Hoerdt, Requirements for discovery of dynamic SSM Sources, IETF, Internet Draft - work in progress 00, February 2005. [Online]. Available: ftp.rfceditor.org/in-notes/internet-drafts_ draft-lehtonen-mboneddynssm-req-00.txt

[10] Romdhani, M. Kellil, H.-Y. Lach, A. Bouabdallah, H. Bettahar, IP Mobile Multicast: Challenges and Solutions, IEEE Comm. Surveys \& Tutorials, 6, 1, 18-41 (2004).

[11] T. C. Schmidt and M. Wählisch, Seamless Multicast Handover in a Hierarchical Mobile IPv6 Environment (M-HMIPv6), individual, Internet Draft - work in progress 03, April 2005. [Online]. Available: ftp: //ftp.rfc-editor.org/in- notes/ internetdrafts/draft-schmidt-waehlisch-mhmipv6-03.txt

[12] B. Fenner, B. Haberman, H. Holbrook, I. Kouvelas, Multicast Source Notification of Interest Protocol, IETF, Internet Draft - work in progress (expired) 05, March 2004.

[13] R.-S. Chang and Y.-S. Yen, A Multicast Routing Protocol with Dynamic Tree Adjustment for Mobile IPv6, Journ. Information Science and Engineering, 20, 1109-1124 (2004).

[14] Romdhani, M. Kellil, H.-Y. Lach, A. Bouabdallah, H. Bettahar, Mobility - Aware Rendezvous Point for Mobile Multicast Sources, in Proceedings of WWIC 2004, ser. LNCS, P. L. et. al., Ed., Berlin Heidelberg: Springer-Verlag, 2957, 62-73 (2004).

[15] T. C. Schmidt and M. Wählisch, Analysis of Handover Frequencies for Predictive, Reactive and Proxy Schemes and their Implications on IPv6 and Multicast Mobility, in Networking - ICN 2005, ser. LNCS, P. Lorenz and P. Dini, Eds.,. Berlin Heidelberg: Springer Verlag, 3421, 1039-1046 (2005).

[16] D. Thaler, Supporting Mobile SSM Sources for IPv6 (MSSMSv6), Individual, Proceedings of IETF Meeting, December 2001. [Online]. Available: www.ietf.org/ proceedings/01dec/slides/magma- 2.pdf

[17] C. Jelger and T. Noel, Supporting Mobile SSM sources for IPv6 (MSSMSv6), individual, Internet Draft - work in progress (expired) 00, January 2002.

[19] B. Fenner, M. Handley, H. Holbrook, I. Kouvelas, Protocol Independent Multicast - Sparse Mode (PIM-SM): Protocol Specification (Revised), IETF, Internet Draft - work in progress 11, October 2004.

[19] T. C. Schmidt and M. Wählisch, A First Performance Analysis of the Tree Morphing Approach to Source Mobility in Source Specific Multicast Routing, Peoceedings of the IEEEE ICN'06 (Pascal Lorenz, Petre Dini ed.), IEEE Press, Aprl 2006.

[20] H. Soliman, Mobile IPv6. Boston: Addison-Wesley, 2004. 


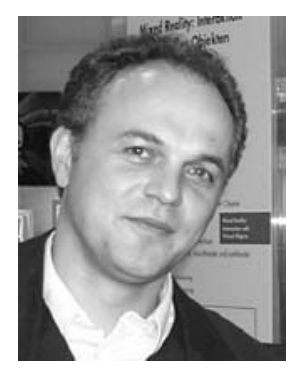

Thomas C. SCHMIDT is professor of Computer Networks and Information Engineering at the HAW Hamburg and project manager at FHTW Berlin, where he was head of the computer centre for many years. He studied mathematics and physics at Freie Universität Berlin and University of Maryland, USA. Since the late 1980s he has been involved in many computing projects, focusing on simulation and parallel programming and distributed information systems. His current fields of interest lie in the areas of next generation Internet (IPv6), mobile and multimedia networking and hypermedia information processing, where he has continuously conducted numerous projects on national and international level.

MatTHIAS WÄHLISCH is a member of the networking group at the computer centre of FHTW Berlin. He is a student of computer science and new german literature at Freie Universität Berlin. His major fields of interest lie in networking protocols, mobile and QoS networks as well as network management, where he looks back on six years of professional experience in project work and publication. 